IdeAs

Idées d'Amériques

$5 \mid 2015$

Crise ou transformations du monde du travail dans les

Amériques

\title{
L'Impossible Syndicalisation. Mobilisations syndicales et répressions dans les zones franches d'exportation du Guatemala (1990-2010)
}

An Impossible Labor Process? Labor Mobilizations and Repressions in Export-

Processing zones in Guatemala (1990-2010)

Una organización sindical imposible. Movilizaciones laborales y represión en las zonas francas de Guatemala (1990-2010)

\section{Quentin Delpech}

\section{(2) OpenEdition}

Journals

Édition électronique

URL : https://journals.openedition.org/ideas/983

DOI : $10.4000 /$ ideas.983

ISSN : 1950-5701

Éditeur

Institut des Amériques

\section{Référence électronique}

Quentin Delpech, «L'Impossible Syndicalisation. Mobilisations syndicales et répressions dans les zones franches d'exportation du Guatemala (1990-2010) », IdeAs [En ligne], 5 | 2015, mis en ligne le 05 juin 2015, consulté le 20 octobre 2022. URL : http://journals.openedition.org/ideas/983 ; DOI : https:// doi.org/10.4000/ideas.983

Ce document a été généré automatiquement le 20 octobre 2022

\section{cc)}

Creative Commons - Attribution - Pas d'Utilisation Commerciale - Pas de Modification 4.0 International - CC BY-NC-ND 4.0

https://creativecommons.org/licenses/by-nc-nd/4.0/ 


\title{
L'Impossible Syndicalisation. Mobilisations syndicales et répressions dans les zones franches d'exportation du Guatemala (1990-2010)
}

\author{
An Impossible Labor Process? Labor Mobilizations and Repressions in Export- \\ Processing zones in Guatemala (1990-2010) \\ Una organización sindical imposible. Movilizaciones laborales y represión en las \\ zonas francas de Guatemala (1990-2010)
}

\section{Quentin Delpech}

\section{Introduction}

1 Cet article tente de cerner les obstacles d'une part à la syndicalisation et d'autre part à la consolidation des organisations syndicales dans les usines de l'habillement du Guatemala, appelées maquilas, qui produisent pour des marques de prêt-à-porter du Nord. La thématique des droits des salariés de ce type d'usines du Sud (export processing zones) a suscité de nombreuses recherches académiques (Anner M., 2009; ArmbrusterSandoval R., 2005 ; Bandy J., 2004 ; Williams H., 1999 ; Frundt H., 2005 ; Carty V., 2004) ainsi que de nombreuses publications de la part de l'Organisation internationale du Travail (OIT) (Gopalakrisnan R., 2007) ${ }^{1}$. Ces travaux ont mis en évidence la violence des relations de travail (les mauvais traitements de la part des superviseurs, les discriminations sexuelles et ethniques, les humiliations, le harcèlement sexuel), l'emprise du contrôle hiérarchique et de la discipline au travail, les conditions d'hygiène et de sécurité déplorables, les violations des droits au travail (les heures supplémentaires obligatoires, l'exploitation, par des salaires souvent tronqués, par les 
extorsions ou les calculs abusifs des directions, les licenciements irréguliers, de femmes enceintes notamment, etc.). Ces recherches se sont par ailleurs intéressées aux obstacles multiples à l'action collective de salariés des usines du Sud en soulignant les stratégies d'anticipation et de contournement mises en œuvre par les employeurs locaux à l'égard des mobilisations collectives et en mettant en exergue le rôle des autorités locales dans la domestication et la répression de ces mobilisations (Williams H., 1999 ; McKay S., 2001, 2006).

2 Au Guatemala, depuis le début des années 1980, l'industrie de l'habillement a connu un développement exponentiel à travers l'attraction massive de capitaux étrangers dans le sillage d'accords de libre-échange (Gereffi G., 2000). Ces usines, appelées maquilas ou maquiladoras, sont devenues au cours des années 1990 l'une des principales sources d'emploi formel dans le pays. Au Guatemala, entre 1984 et 2000, le nombre de maquilas est ainsi passé de 41 à 784 employant désormais plus de cent mille travailleurs. Ces chiffres permettent de prendre la mesure d'un phénomène - nommé par certains " maquiladora revolution» (Petersen K., 1992) - souvent présenté comme un processus d'industrialisation accéléré, d'ouverture du marché guatémaltèque au temps du capitalisme global.

Cet article cerne les obstacles pratiques et les contraintes quotidiennes à l'exercice des droits syndicaux des salariés. Ce travail se fonde sur un travail d'enquête approfondie, mené entre 2006 et 2009, dans quatre usines de l'habillement de la proche périphérie de Guatemala Ciudad où ont émergé depuis le début des années 2000 quatre syndicats d'employés. La méthode d'enquête a alterné le recours à des sources de première main (des entretiens semi directifs - des syndicalistes, des directeurs de ressources humaines, des avocats et des fonctionnaires - mais aussi des discussions informelles avec ces mêmes enquêtés) et de seconde main à travers le recours à des sources judiciaires, journalistiques et administratives ainsi que des études d'organismes spécialisés (OIT). Une trentaine d'employés de ces usines ont été interviewés ainsi que des syndicalistes, des avocats, des activistes, des inspecteurs du travail, etc. Afin de fournir une approche des obstacles concrets à l'exercice des droits syndicaux et de négociation collective dans les maquilas du Guatemala, on procédera en premier lieu à l'examen des contraintes à la syndicalisation, puis à l'analyse des répressions des organisations syndicales.

\section{Des obstacles multiples à la syndicalisation}

4 Le Guatemala présente l'un des taux d'affiliation syndicale le plus faible d'Amérique latine: seulement $3 \%$ de la population active serait syndiquée et, dans de très nombreux secteurs économiques, la syndicalisation reste un phénomène extrêmement limité. Les $m$ aquilas de l'habillement, installées dans des zones franches d'exportation, et dont le nombre n'a cessé d'augmenter à partir des années $1980^{2}$, offrent un exemple d'un secteur d'activité caractérisé par sa très faible syndicalisation. Depuis les débuts de l'industrie, très peu d'organisations syndicales ont vu le jour dans l'industrie. Ces dix dernières années, seulement quelques syndicats ont émergé et seulement quatre sont parvenus à développer une activité syndicale relativement pérenne même si les autorités du ministère du Travail au Guatemala revendiquent un nombre plus important d'organisations dans le secteur ${ }^{3}$. 
Plusieurs facteurs expliquent les difficultés d'émergence d'organisations syndicales dans le secteur des industries globales de l'habillement au Guatemala. On peut distinguer différents facteurs qui rendent compte de la très faible syndicalisation dans les maquilas de ce pays: des logiques liées aux propriétés de la main-d'œuvre, des logiques propres à l'activité d'organisation des fédérations syndicales nationales, des logiques liées aux stratégies antisyndicales des employeurs et, enfin, des logiques relatives à la déficience des institutions étatiques au Guatemala.

\section{Une main d'œuvre peu qualifiée et précaire}

6 De très nombreuses recherches ont mis en avant le fait que les faibles ressources matérielles et sociales des salariés des maquilas les éloignent de la possibilité d'une participation à une activité syndicale (Pena D., 1997; Perez Sainz J.-P., 2000, 2007 ; Perez Sainz J.-P., Leal A., 1992 ; Williams H., 1999). En effet, la précarité des salariés, le plus souvent des femmes, rend ces dernières dépendantes de leur emploi quand bien même elles dénonceraient les abus qu'elles vivent sur le lieu de travail. Face à la prégnance de l'informalité, l'accès au salariat que représentent les emplois dans les maquilas reste le « choix le moins pire ». Aussi, le risque que représente la participation à la formation d'un syndicat est souvent trop important au regard de cette dépendance. C'est du moins l'un des arguments régulièrement évoqués par les salariés pour expliquer leur non engagement dans des organisations syndicales : la peur de perdre leur emploi. Cette dépendance tient tout d'abord à la précarité des salariés, une précarité professionnelle, salariale et sociale.

\section{Des salariés pauvres dépendants de leur emploi}

7 D'après l'Enquête nationale sur l'emploi et les revenus (ENEI, 2010), 75,4 \% de la population active du Guatemala travaille dans des activités informelles. L'économie informelle est ainsi la «norme » de l'emploi au Guatemala. Les activités informelles recouvrent un ensemble d'activités liées au commerce, à l'artisanat, aux services. Pour de nombreux employés de maquila, l'accès au salariat industriel représente une échappatoire aux formes précaires et peu sécurisées des activités informelles mais engendre également une réorganisation de l'économie des foyers, dans une industrie dont la main-d'œuvre est fortement féminisée. Sans disposer de données précises sur la main-d'œuvre des usines métropolitaines du Guatemala ${ }^{4}$, on peut néanmoins souligner des trajectoires représentatives de ce que l'accès à l'emploi dans la maquila a changé dans l'économie familiale. On peut tout d'abord constater que le travail dans le secteur des maquilas est souvent corrélé à la précarité de l'emploi masculin au sein du foyer. Nombre de travailleuses enquêtées évoquent cette situation: la recherche de l'emploi dans les maquilas serait liée à la nécessité de rééquilibrer l'insécurité des emplois des maris ou conjoints ou, dans d'autres cas, la nécessité de travailler suite à une rupture au sein du couple ou de la famille. De manière corrélative, pour de nombreux hommes employés dans la maquila, ce travail incarne une certaine sécurité au regard d'expériences précaires sur le marché de l'emploi, dans la construction notamment. On retrouve par ailleurs des situations très diffuses d'employés (homme ou femme), le plus souvent jeunes, dont l'entrée dans le secteur des maquilas et l'accès au salariat permet d'aider l'ensemble de la structure familiale. Dans la majorité des cas, ces jeunes n'ont pas d'autres expériences de travail. 
Cette dépendance à l'emploi dans les maquilas est renforcée depuis 2004 notamment par la crise que connaît le secteur. Depuis 2004, le nombre d'employés de l'industrie ne cesse de diminuer, à l'exception d'une légère augmentation en 2010. Selon les organisations patronales, cette diminution de l'emploi dans le secteur des maquilas est liée à la concurrence internationale et à la perte de compétitivité de l'industrie guatémaltèque. De très nombreuses maquilas ont ainsi fermé leurs portes à partir de cette date. Les employeurs du secteur évoquent différents facteurs pour expliquer cette diminution de l'emploi : la concurrence des usines chinoises, les différences des coûts du travail vis-à-vis des autres pays centraméricains, le coût élevé de l'énergie électrique, etc. Cette fragilité de l'emploi contraste avec la période de croissance des années 1990 et du début des années 2000, qui constitue d'une certaine manière l'apogée de l'industrie. À cette période, le secteur emploie plus de 120000 salariés uniquement pour le travail de confection. Pour autant, ces chiffres ne prennent pas en compte les emplois liés à l'activité des maquilas, c'est-à-dire l'ensemble des services allant de l'activité liée à la fourniture de textile au transport en passant par les activités de soustraitance liées à la marchandisation des produits finaux. En 2004, les produits de l'habillement représentent $33 \%$ de la valeur des exportations du pays, loin devant les produits traditionnels d'exportation que sont le café, le sucre ou les bananes (PNUD, 2010 :133). Comme l'indiquent deux employés de maquila, l'accès à l'emploi dans le secteur est, depuis le milieu des années 2000, plus compétitif et contraste avec une époque où un travail pouvait être trouvé du jour au lendemain :

- César (26 ans, huit années d'ancienneté, originaire de Mixco) : «Y a un moment, au début, à la fin des années 1990 peut-être, trouver un emploi à la maquila, c'était facile. C'était le paradis, moi je me permettais même de démissionner la veille et le lendemain, je trouvais un emploi comme ça. Aujourd'hui, travailler à SAE [la maquila dans laquelle il travaille] c'est devenu difficile, tout le monde veut travailler ici. Avant, c'était facile de trouver un emploi, y avait des annonces, des pancartes, on s'y rendait et puis on commençait tout de suite, sans savoir rien du boulot. »

- Gloria (39 ans, vingt années d'ancienneté, originaire de Sayaxché): « À l'époque, tu trouvais du travail facilement, moi j'ai entendu parler d'un emploi, j'y suis allée et j'ai travaillé directement. Bon ça ne m'a pas plu, je suis partie et j'ai retrouvé un travail rapidement. Aujourd'hui c'est plus comme ça. Ils ne demandaient rien avant, tu venais travailler comme ça, sans expérience. »

10 Aussi, les démissions de la part d'employés, facilitées par l'abondance de travail dans le secteur, semblent se raréfier depuis le début des années 2000. En effet, ces solutions d'exit sont rendues coûteuses par la concurrence sur le marché du travail et les rares options que celui-ci offre. Les nombreuses fermetures d'usines ainsi que l'emprise de l'économie informelle entravent les choix d'exit. Aussi, on observe un rétrécissement des possibilités de choix sur le marché du travail dans les maquilas. Alors qu'à un moment, l'abondance d'offres d'emploi permettait aux salariés de démissionner face à des situations conflictuelles au travail par exemple, ce rétrécissement favorise la dépendance des employés à leur emploi et à la nécessité de le conserver.

\section{Des conditions salariales qui renforcent la dépendance des salariés à leur emploi}

11 La précarité des salariés est aussi une précarité salariale. Depuis l'accord gouvernemental 625-2007 de décembre 2007, le président de la République, Oscar Berger, a fixé un salaire minimum spécifique pour les industries d'exportation sous le 
régime du décret 29-89 relatif à la promotion des industries de maquila à un niveau inférieur aux autres types d'activités économiques. Les salariés de maquila se retrouvent ainsi discriminés par une décision de l'État justifiée par la nécessité de rendre l'industrie de l'habillement et du textile au Guatemala plus compétitive. En plus de recevoir seulement un salaire minimum, dont on verra ensuite qu'il ne permet pas aux salariés d'accéder aux besoins de base quotidiens, l'État discrimine les employés de maquila par rapport à d'autres activités agricoles ou industrielles, comme l'indique le tableau suivant :

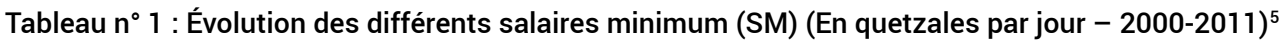

\begin{tabular}{|l|l|l|l|}
\hline Année & SM - activité agricole & SM - activité non agricole & SM - activité de maquila \\
\hline 2011 & 63,70 & 63,70 & 59,45 \\
\hline 2010 & 56,00 & 56,00 & 51,75 \\
\hline 2009 & 52,00 & 52,00 & - \\
\hline 2008 & 47,03 & 48,50 & 47,75 \\
\hline 2007 & 44,85 & 45,82 & \\
\hline 2006 & 42,46 & 43,64 & \\
\hline 2005 & 38,60 & 39,67 & \\
\hline
\end{tabular}

\section{Source : Ministère du Travail, Banco de Guatemala}

L'analyse du ratio entre le salaire minimum dans le secteur des maquilas et l'accès aux besoins essentiels mesurés ici par deux indicateurs différents du coût de la vie (canasta básica vital $-\mathrm{CBV}^{6}$ et canasta básica alimentaria $-\mathrm{CBA}^{7}$ ) montre bien comment les salaires des travailleurs de maquila ne comblent pas les dépenses minimales en termes d'alimentation notamment.

Tableau $n^{\circ} 2$ : Salaire minimum dans le secteur des maquilas et accès aux besoins vitaux (En quetzales par mois - 2000-2009)

\begin{tabular}{|l|l|l|l|}
\hline Année & $\begin{array}{l}\text { Canasta básica vital (Panier } \\
\text { VITAL DE BASE) }\end{array}$ & $\begin{array}{l}\text { Canasta básica alimentaria (PANIER } \\
\text { ALIMENTAIRE DE BASE) }\end{array}$ & $\begin{array}{l}\text { Salaire } \\
\text { minimum }\end{array}$ \\
\hline 2000 & 2121.37 & 1162.51 & 715.50 \\
\hline 2005 & 2564.83 & 1405.53 & 1190.10 \\
\hline 2006 & 2724.94 & 1493.27 & 1309.20 \\
\hline 2007 & 3033.92 & 1662.59 & 1374.50 \\
\hline 2008 & 3605.94 & 1976.05 & 1455.00 \\
\hline
\end{tabular}




\begin{tabular}{|l|l|l|l|}
\hline 2009 & 3462.26 & 1897.32 & 1560.00 \\
\hline
\end{tabular}

Source : PNUD, Rapport national 2010 sur le développement humain, Guatemala

13 Aussi, quand bien même l'accès à l'emploi dans une maquila reste une opportunité de sécurité face à l'omniprésence de l'informalité, les rétributions financières des employés de maquila les conditionnent à des modes de vie difficiles. Sachant que, bien souvent, les salaires alimentent l'économie de l'ensemble des foyers, le quotidien des salariés est ainsi marqué par le manque et la primauté du nécessaire.

\section{Des salariés peu qualifiés méconnaissant leurs droits}

14 La précarité des employés est enfin liée à leurs faibles ressources scolaires et professionnelles. Les enquêtes réalisées auprès de salariés de maquila soulignent la faiblesse de leur capital scolaire. Une majorité d'entre eux ne disposeraient que d'un niveau basique de scolarisation, une majorité a terminé les années d'éducation primaire et, dans une moindre mesure, les années dites de "cycle basique ", d'études secondaires. À la recherche d'une main-d'œuvre peu qualifiée, les maquilas offrent ainsi un débouché pour une population marquée par des trajectoires scolaires courtes. Selon une étude réalisée sur des employées de maquilas et d'activités agroalimentaires de la région de Chimaltenango qui représentent environ $85 \%$ de la main-d'œuvre locale, seul un tiers d'entre elles savent lire et écrire, $43,9 \%$ ont suivi le cycle primaire et seules 12,8 \% le cycle basique (jusqu'à 15 ans) (Médecins du Monde, 2010). Ces caractéristiques sociales ont des effets sur les modalités de précarisation au travail. En effet, de nombreuses employées sont recrutées uniquement suite à un accord oral, sans signer de contrat écrit. Cette situation peut ainsi aboutir à faciliter leur licenciement. Ce type de situation reste néanmoins minoritaire mais, de manière générale, la majorité des employés des maquilas du Guatemala méconnaît les droits que leur confère leur situation de travailleurs salariés. Le faible capital scolaire des employés de maquilas renforce ainsi leur vulnérabilité au travail. Voilà la manière dont trois salariés de maquila interviewés évoquent leur méconnaissance de leurs droits :

- Gloria : «Moi avant je savais rien de tout ça. Les droits, le Code du travail, le salaire, je savais pas à quoi on avait droit. Par exemple, les bonus de production, c'est toujours compliqué de savoir, de comprendre, parfois les superviseurs augmentent les objectifs comme ça, ils n'ont pas le droit. »

- Adriana (36 ans, quinze années d'ancienneté, originaire de Villa Nueva) : «Au début, je ne savais pas à quoi on avait droit. Maintenant, je comprends mais au début, par exemple le Code du travail, je ne connaissais pas alors les horaires, on savait pas comment ça devait fonctionner, qu'on avait des droits, qu'une journée de travail c'est... euh... règlementé. »

- César : « La connaissance des droits, c'est venu au fur et à mesure, mais au début, c'est vrai qu'on a qu'une idée vague de nos droits, sur les salaires par exemple, les indemnités aussi.»

De plus, les faibles qualifications des salariés favorisent les pratiques de turn over. L'une des invectives récurrentes des contrôleurs de chaînes de montage à l'encontre des salariés consiste à nier la subjectivité des salariés en niant l'utilité des ouvriers : «ce travailleur ne sert à rien » (« este trabajador no sirve »). Pour autant, les salariés ne servent 
d'autant plus à rien qu'ils peuvent être remplacés et qu'ils sont perçus comme interchangeables par l'encadrement. Les travailleurs sont interchangeables à la fois car il existe une disponibilité de travail sur un marché de l'emploi marqué par des formes précaires et informelles de subsistance mais aussi parce que, en tant qu'emploi non qualifié, le travail dans la maquila n'implique pas de la part de la direction la prise en compte des salariés, ni de ce qu'ils ressentent. Les pourcentages relatifs à l'ancienneté des travailleurs dans trois maquilas de Mixco, restitués dans le tableau suivant, en témoignent :

Tableau $n^{\circ} 3$ : Répartition par ancienneté des travailleurs dans l'industrie du textile et de l'habillement (Industrie - par usine) ${ }^{8}$

\begin{tabular}{|l|l|l|l|}
\hline $\begin{array}{l}\text { ancienneté } \\
\text { usine }\end{array}$ & moins de 2 ans & entre 3 et 5 ans & plus de 5 ans \\
\hline $\begin{array}{l}\text { SAE-A International S. A. } \\
\text { (Planta A) }\end{array}$ & $39,0 \%$ & $37,8 \%$ & $26,4 \%$ \\
\hline $\begin{array}{l}\text { SAE-A International S. A. } \\
\text { (Planta C) }\end{array}$ & $44,3 \%$ & $37,8 \%$ & $19,0 \%$ \\
\hline
\end{tabular}

Source : Registre des entreprises SAE-A International S. A. et Winners S. A.

On le voit bien, une minorité de salariés reste plus de cinq années dans les entreprises. Cette situation est liée aux pratiques de turn over de la direction des entreprises qui permettent d'exercer encore plus de pression sur les employés en leur suggérant qu'ils sont interchangeables.

\section{Une main d'œuvre peu ajustée aux stratégies de mobilisation des fédérations nationales}

18 Une autre contrainte à la syndicalisation tient cette fois à l'activité de mobilisation des centrales et fédérations syndicales nationales. Une première difficulté que rencontrent les syndicalistes des fédérations dans leur tentative d'organisation des employés des maquilas concerne leur incapacité à s'ajuster à la problématique de la féminisation de la main-d'œuvre industrielle. Les syndicalistes des fédérations ne disposent pas de stratégies d'organisation susceptibles de mobiliser efficacement les employées qui sont majoritaires dans l'industrie; le cœur de leur travail de mobilisation restant associé aux secteurs publics et industriels (agro-industrie notamment, cimenterie, etc.) dominés par une main-d'œuvre masculine. De plus, la création d'un «comité de femmes » au sein des structures syndicales au niveau national n'a pas permis un réel renouvellement des postes et une accession des femmes syndicalistes au sein des fédérations. De ce fait, certaines problématiques clés pour la syndicalisation des employées de maquilas (dignité, problèmes des licenciements abusifs et de discrimination à l'embauche de femmes enceintes, harcèlement sexuel sur le lieu de travail) ne sont pas mobilisées par les organisateurs au niveau national. D'autre part, lorsqu'une organisation syndicale finit par émerger, les problématiques de genre sont souvent délaissées au profit des enjeux salariaux. 


\section{La peur de s'organiser} de s'organiser. Dans leur grande majorité, s'organiser rime avec un risque inconsidéré de perdre son emploi. Cette peur est notamment le résultat de la diffusion d'un message de la part des employeurs du secteur selon lequel l'émergence d'un syndicat serait un risque pour les emplois. De nombreux exemples montrent que les employeurs utilisent différents arguments pour alimenter cette peur : le fait qu'un syndicat risque de faire augmenter le coût du travail, de faire baisser la compétitivité de l'entreprise, et donc de perdre des commandes de clients. De plus, l'idée de se syndiquer trouve rarement l'assentiment des familles. De nombreux syndiqués expliquent que leur engagement dans un syndicat n'est pas accepté au sein de leur famille qui évoque leur peur :

20 - Enquêteur : «Que pensaient vos enfants et votre compagnon du syndicat?»

- Gloria « Ils n'étaient pas d'accord avec cette idée, il [son compagnon] me disait que c'était très risqué. Avant, il connaissait des cas de syndicalistes qui avaient été tués ou séquestrés... Il ne voulait pas. Et mes enfants même s'ils ne connaissaient pas le syndicat, ils ne voulaient pas non plus. Ils avaient peur. »

- César : « Le syndicat c'est comme un péché mortel pour certains [...] Si tu te mets dans un syndicat, ils vont te tuer... c'est ce que pensent beaucoup de gens. »

21 - Enquêteur : «Des travailleurs disaient ça?»

- César : «Mon père et ma mère ont peur, aujourd'hui peut-être moins parce qu'ils se sont habitués mais au début oui... Ils ont même changé de chambre [rires]. Ma mère au début elle était vraiment contre, maintenant elle s'habitue, souvent elle me dit de sortir du syndicat, que je n'y gagne rien. Ils me disent qu'un jour il va m'arriver quelque chose."

- Enquêteur : « Ils disaient quoi votre mari de ça ?»

- Margarita : «Euh... Je suis désolée d'être vulgaire mais il me disait de sortir de cette merde, qu'on allait me tuer ou le tuer et qu'est-ce qu'on allait faire des enfants... Oui... Il n'a jamais été d'accord. »

\section{Des pratiques patronales de contournement et d'anticipation des organisations syndicales}

D'autres obstacles à la syndicalisation des employés de maquila relèvent davantage de stratégies de la part des employeurs qui visent à anticiper l'émergence d'organisations syndicales sur le lieu de travail. Ces derniers contournent les règles légales pour empêcher la formation de syndicats. Parmi les lois et décrets qui régissent l'activité des maquilas/maquiladoras au Guatemala figure le décret 29-89 (Ley de fomento y desarrollo de la actividad exportadora y de maquila). Ce décret réglemente notamment les bénéfices dont peuvent jouir les entreprises soumises au régime spécifique déterminé par cette loi. Ainsi, selon les termes du décret, ces entreprises disposent de différentes exonérations fiscales et notamment une exonération totale de l'impôt sur le revenu pendant une période de dix ans. Selon certains chiffres, l'ensemble des exonérations fiscales autorisées par l'État pour les activités économiques représentait en 2004 près de 26 milliards de quetzales, soit environ 12,3\% du produit intérieur brut (PIB) (CIIDH, 
2006). De ce total, les exonérations du décret 29-89 sont estimées à trois milliards de quetzales, soit plus d'1\% du PIB annuel. Ces exonérations sont justifiées par une stratégie d'attraction des investissements étrangers et nationaux et de promotion du développement du commerce extérieur au Guatemala. L'autorisation, la supervision et le contrôle du régime spécifique relatif au décret 29-89 dépendent du Ministère de l'Économie et des services fiscaux de l'État. Ce régime fait néanmoins l'objet de nombreuses fraudes et de contournements de la part des employeurs qui sont tolérés par les institutions d'État (Delpech, 2014).

Une autre pratique patronale très répandue concerne le contournement du régime d'exonération. Cette fraude a néanmoins des effets sur la possibilité d'organiser les lieux de production. L'une des pratiques frauduleuses les plus répandues parmi les entreprises d'exportation - et notamment dans les entreprises maquilas - consiste à contourner la limitation d'exonération d'impôts. Au bout des dix ans de bénéfices, les entreprises ferment en se déclarant en faillite et se reforment sous un autre nom et dans un autre endroit afin de pouvoir jouir à nouveau de la période d'exonération. Ces fermetures illégales, dans le sens où elles ne sont pas motivées par de réels arguments économiques mais par des stratégies de contournement du régime des maquilas, sont difficiles à quantifier de manière précise. Deux types de sources peuvent néanmoins nous permettre de mieux saisir la banalisation de ces pratiques. En premier lieu, des sources statistiques qui restent néanmoins très difficiles à manier. Les chiffres relatifs aux fermetures de maquilas donnés par l'organisation VESTEX, organisation patronale sectorielle de l'habillement, soulignent la baisse importante de la main-d'œuvre employée depuis 2004. Selon VESTEX, en l'espace de quatre années, près de 50000 emplois auraient disparu et un nombre important de maquilas auraient fermé (VESTEX, 2010). Il est pourtant impossible de distinguer les fermetures illégales et légales. Les chiffres de l'inspection du travail témoignent eux aussi d'une augmentation du nombre de fermetures d'usines, mais sans préciser si ces fermetures aboutissent à des réouvertures et des changements d'employeurs liés à une stratégie de contournement du régime des maquilas. Les entretiens réalisés, ainsi que certains rapports officiels, renseignent sur ces pratiques frauduleuses qui sont connues des salariés, soit par l'expérience vécue, soit par la circulation d'informations via des collègues de travail, des proches ou des voisins.

24 Nombreux sont les syndicalistes qui considèrent même qu'il est impossible d'organiser les salariés du secteur. Pour eux, toute mobilisation serait de fait à «durée déterminée » en raison des pratiques illégales de fermeture, comme l'affirme cette syndicaliste guatémaltèque :

25 - Irene Barrientos : « Mais, le problème réside en fait dans la loi de zones franches des maquilas, ils ont des périodes d'exonération, c'est-à-dire pas d'impôts et ceci pendant dix ans. Mais les maquilas... La majorité des maquilas au Guatemala ont plus de dix ans mais ils continuent de ne pas payer d'impôts et pourquoi? Justement par cette possibilité [changer de nom, de lieu de production]. Une maquila qui s'appelle aujourd'hui... euh...X [nom d'une maquila], avant d'atteindre les dix ans, elle change de nom et de site et ils font cela comme un cycle, recouvrant dix ans de période d'exonération. Alors qu'est ce qui se produit? Ils ne génèrent pas de revenus [pour l'État] parce qu'ils ne paient pas d'impôts, ils paient mal les travailleurs, les maltraitent physiquement, moralement. Ça fait de nombreuses conditions très peu favorables à l'émergence de syndicats dans les maquilas. En plus, dans le cadre des négociations des 
accords de paix, nous, les organisations syndicales, on a beaucoup discuté, notamment autour d'un pacte fiscal au Guatemala, on s'est donné comme principe de favoriser une régulation par l'Etat... Une régulation de l'activité maquiladora du pays, et de créer une loi qui les obligerait à payer des impôts. Alors, ils ont commencé à menacer d'aller au Nicaragua, de quitter le Guatemala parce que justement le Nicaragua avait encore des avantages fiscaux. Ça c'est le jeu qu'utilisent les entrepreneurs des maquilas et ce jeu maintient la situation et... Il nous tient nous aussi... Les travailleurs. »

Plus qu'une exonération fiscale, le contournement de la loi permet aux employeurs de s'exonérer du respect des droits sociaux à l'égard de la main-d'œuvre. Ces fraudes tolérées par l'État constituent par ailleurs une manière de se "débarrasser » de la main-d'œuvre en toute impunité. Ces pratiques ont néanmoins des conséquences sur la possibilité d'émergence d'organisations syndicales. Les pratiques de segmentation des usines/lieux de production - créer des sociétés anonymes / pour jouir des périodes de défiscalisation par exemple - et de recours à des sous-traitants - ne sont pas uniquement motivées par un souci économique. Cette segmentation recherchée pour des raisons fiscales a aussi un intérêt stratégique pour les employeurs dans leur lutte contre de possibles formes d'organisation sur le lieu de travail. De manière concrète, les employeurs créent différentes sociétés anonymes pour réaliser les commandes. L'exemple d'une entreprise sud-coréenne montre que celle-ci prétend être propriétaire de sept usines produisant au Guatemala. Or, toutes ces usines ont des noms différents, bien qu'appartenant à la même maison mère. Le fait de segmenter les centres de travail en sociétés anonymes permet en réalité d' "éteindre » rapidement des protestations ou des tentatives de syndicalisation en fermant des sites, sans que la production ne soit trop affectée. Les employeurs utilisent ainsi ces pratiques de segmentation à la fois pour couper court à de possibles formes d'organisation des salariés et éviter des phénomènes de diffusion. Ces pratiques s'accompagnent, selon de nombreux salariés ou syndicalistes, d'une circulation de «listes noires" de salariés syndiqués entre les différents employeurs du secteur afin d'empêcher le recrutement ou parfois la réintégration d'éléments perçus comme perturbateurs.

De plus, le recours à la sous-traitance dans de plus petites usines est par ailleurs très développé au Guatemala. Selon les employeurs, ces pratiques d'externalisation se justifient par la nécessité de réaliser les commandes dans des délais contractuels, sous peine de paiement de pénalités et donc de baisse des marges. Le recours à la soustraitance peut aussi viser à affaiblir une organisation syndicale ou à discipliner une main-d'œuvre au sein de laquelle des velléités d'organisation ont été identifiées: fermer temporairement les opérations d'une usine pour répondre à certaines commandes.

\section{Des pratiques illégales de licenciements antisyndicaux}

Les employeurs disposent par ailleurs d'un autre moyen de mettre définitivement fin à de possibles mobilisations sur le lieu de travail: les licenciements du personnel soupçonné de s'organiser.

- Enquêté (chef de production d'une maquila) : «Au Guatemala, dans le secteur [des maquilas], la syndicalisation est difficile, en plus de ça, une entreprise de maquila, quand elle localise un foyer de syndicalisation [foco sindical], elle les licencie rapidement. L'entreprise licencie les leaders. [...] Moi ça m'est arrivé une fois, dans une entreprise 
où je travaillais avant, il $\mathrm{y}$ avait une personne qui, juste pour avoir dit qu'elle allait amener un syndicaliste, a été licenciée. [...]»

- Enquêteur : «Qui s'en occupe de ça?»

- Enquêté : «C'est au niveau de la direction, y'a différents départements, et là c'est la direction, l'information... Enfin, la rumeur c'est les superviseurs qui la donnent, je me souviens même moi j'avais été entendu dans cette affaire. »

Comme l'indique cet ancien chef de production d'une maquila, ce type de pratiques consistant à localiser des «foyers" de contestation au sein de la main-d'œuvre est largement développé au Guatemala9. Il est difficile de cerner de manière statistique ces pratiques, néanmoins, les entretiens réalisés avec les salariés soulignent dans quelle mesure cette pratique relève de l'ordinaire. La majorité des enquêtés disent en avoir entendu parler, ou de l'avoir vu se produire sur leur lieu de travail. De manière générale, les violations des droits syndicaux au Guatemala les plus régulièrement dénoncées dans les plaintes auprès du Comité de la liberté syndicale concernent le licenciement d'affiliés ou de dirigeants syndicaux.

\section{Des institutions de l'État déficientes}

De manière générale, comme l'atteste l'exemple des fraudes tolérées par les services de l'État, l'inaction des autorités étatiques contribue à renforcer ces obstacles multiples à la syndicalisation. Les organes de contrôle de l'OIT ont ainsi très souvent mis en avant le fait que la déficience des institutions favorisait un climat d'impunité lui-même favorable à la non-syndicalisation au Guatemala. Cette problématique est à mettre en lien avec les moyens humains et matériels des institutions chargées de contrôler les normes du travail au Guatemala. De nombreux éléments montrent que l'inaction de certaines institutions de l'État tient à leur faiblesse budgétaire tout d'abord. Des rapports et des commentaires d'organisations syndicales (OIT, 2009) ont souligné par exemple les déficiences des services d'inspection du travail: manque de formation professionnelle, carences en termes de matériel (essence des automobiles utilisées par les inspecteurs), manque de personnel (très peu de fonctionnaires réalisent un travail régulier et organisent des inspections sur les lieux de travail, ils tendent à se focaliser sur les tâches purement administratives), problèmes de rémunération (des salaires faibles et des conditions de promotion opaques qui favorisent le risque de corruption), etc. Cette analyse pourrait être réalisée pour l'ensemble des services étatiques liés au contrôle et à la sanction des conflits du travail au Guatemala : les tribunaux, les services d'inspection des conditions d'hygiène et de sécurité, l'inspection de la sécurité sociale, etc.

\section{La répression des organisations syndicales du secteur}

Selon certains travaux, la "durée de vie » moyenne des organisations syndicales dans le secteur n'excèderait pas les trois années (Rodriguez-Garavito C., 2008). Plusieurs facteurs peuvent expliquer cette impossibilité syndicale à pérenniser son action. Les fermetures d'usines tout d'abord utilisées pour mettre un terme aux mobilisations syndicales; en ce sens, la mobilité du capital est un obstacle à la consolidation des organisations syndicales. D'autre part, les pratiques répressives variées rendent difficile l'activité syndicale sur la durée. Le Comité de la liberté syndicale a rappelé à 
plusieurs reprises dans quelle mesure l'exercice des droits syndicaux ne pouvait se développer dans un climat de violence et d'incertitude. Pour autant, le Guatemala partage avec la Colombie les chiffres d'assassinats de militants syndicaux les plus élevés au monde. Entre 2006 et 2011, 53 syndicalistes ont été assassinés au Guatemala (OIT, 2011). L'Organisation internationale du Travail (OIT) souligne la persistance de violences antisyndicales (OIT, 2009). Selon une étude sur les conditions d'exercice des droits syndicaux en Amérique centrale sur la période 1990-2009, près de $20 \%$ des plaintes déposées auprès du Comité de la liberté syndicale de l'OIT concernait des violations relatives à la sécurité et à l'intégrité des syndicalistes, à savoir des menaces de mort, des agressions physiques, des détentions illégales, des homicides, des tentatives de séquestration, des disparitions forcées ou encore des cas de torture. Cette même Organisation dénonce par ailleurs le climat d'impunité qui entoure ces actes. Lors de la dernière Conférence internationale du Travail, la Commission de l'application des normes de la Conférence a parlé d' «impunité grave et quasi totale » en soulignant «la lenteur et l'inefficacité des procédures pénales relatives aux actes de violence, les délais excessifs des procédures judiciaires et [le] manque d'indépendance du pouvoir judiciaire » (OIT, 2011). Victime privilégiée des répressions d'État sous les dictatures des années 1970 et 1980 (Levenson-Estrada, 1994 ; Frundt, 1987), le mouvement syndical continue aujourd'hui de faire l'objet de répressions en dépit d'un processus de "transition démocratique » engagée dès la fin des années 1980. Comment fonctionne concrètement la répression des organisations syndicales dans le secteur des maquilas? Au-delà des cas de violences évoqués et régulièrement dénoncés par les organes de l'OIT et d'autres recherches - licenciements antisyndicaux, violences, harcèlement, listes noires de syndicalistes, menaces verbales et physiques (Gopalakrisnan R., 2007) -, le secteur des maquilas est caractérisé par des formes bien particulières de répression des employés syndiqués, notamment par des formes dissimulées de répression et d'autres pratiques antisyndicales.

\section{Des stratégies de sape antisyndicale}

Les pratiques de discrimination et de répression dans les maquilas du Guatemala sont en partie dissimulées et discrètes. Loin des pratiques bien documentées de violences et de répressions directes, la gestion des organisations syndicales dans les relations professionnelles des maquilas s'apparente à une véritable stratégie de sape antisyndicale. Afin de saper les efforts d'affiliation et d'action syndicales, les employeurs entreprennent notamment de les délégitimer auprès de la main-d'œuvre comme représentant une menace permanente à l'emploi, et par ailleurs d'appliquer des stratégies pour diviser les leaders. Pour les employés syndiqués, l'activité syndicale a de nombreuses conséquences sur leur vie professionnelle et privée, comme nous le verrons plus loin.

\section{Présenter l'activité syndicale comme un péril}

Pour bien saisir les mécanismes à l'œuvre dans les tentatives de délégitimation de l'action syndicale dans les maquilas, il convient de se concentrer sur la construction d'un discours sur la menace que représente le syndicalisme. Pour les employeurs, la revendication de droits n'est pas une action légitime car elle menace la production et par ricochet les emplois, donc la sécurité économique des travailleurs. Cet 
argumentaire se retrouve incarné dans de nombreuses situations où les revendications $d u$ travail s'opposent au droit au travail. Les employeurs mobilisent ainsi l'idée selon laquelle le droit au travail, c'est-à-dire l'accès à l'emploi, primerait sur le droit $d u$ travail comme ensemble de normes juridiques régissant les relations professionnelles. Un extrait de procès-verbal de discussions lors d'une réunion de conciliation entre un syndicat de maquila et les représentants patronaux au sujet du retrait de la mise à disposition de bus pour les ouvriers met en exergue cette opposition :

Représentant de l'entreprise [désigné ci-après «Entreprise »] : «À ce propos, vous n'êtes pas sans savoir que le service de bus a été suspendu pour tous les travailleurs qui vivent à plus de quinze kilomètres de distance [de l'entreprise]. L'entreprise croit savoir que de nombreux travailleurs s'opposent déjà à cette suspension mais elle sera maintenue pour ceux qui vivent à plus de quinze kilomètres de distance et ceux qui s'y opposent feront l'objet d'un licenciement avec indemnités. Mais l'entreprise est certaine qu'ils ne trouveront pas de meilleures conditions de travail que celles dont ils jouissent ici. De plus, il faut prendre en compte le problème de sécurité. Il y a quelques temps l'assassinat d'un travailleur qui se rendait sur son lieu de travail a été perpétré. Il est mort à cause d'une personne qui voulait le voler. Cet événement a ému un membre du comité de direction de l'entreprise car le travailleur ne faisait que tenter de se rendre sur son lieu de travail de manière honnête. Si l'entreprise ferme, alors il y aura plus de chômage et ainsi un risque d'insécurité plus important. Il faut faire des efforts pour que le problème de l'insécurité n'empire pas et pour cela, il faut faire des efforts pour maintenir les emplois des travailleurs [...] D'une certaine manière, il faut analyser ceci avec plus de profondeur pour maintenir les entreprises. Ce qui est demandé [par le syndicat] est juste mais son application mettrait en danger tout le monde, pour cette raison, même si c'est la loi, l'entreprise ne peut pas la respecter ${ }^{10}$."

Cet extrait de discussion souligne comment la précarité économique revendiquée par l'entreprise pousse celle-ci à une recherche de réduction des coûts, processus dans lequel les droits des travailleurs constituent un obstacle. Pour l'entreprise, la revendication des droits met en péril l'entreprise et, par une argumentation bien ficelée, l'emploi, la situation économique et même physique des travailleurs euxmêmes (le " problème de sécurité » et l'évocation du meurtre d'un ouvrier). Le droit au travail s'oppose ainsi aux droits $d u$ travail. Plus exactement, cet extrait montre comment le droit au travail mis en avant par l'entreprise recèle une pensée communément admise au sein du secteur patronal de maquila que l'on pourrait résumer de la sorte: revendiquer est illégitime dans la mesure où l'entreprise fournit les emplois. En d'autres termes, les revendications sont d'autant moins acceptables - et pensables - que l'entreprise, elle, crée l'emploi, c'est-à-dire (toujours selon le mode de pensée du secteur) les conditions du bien-être social des travailleurs. Comment, dès lors, les travailleurs peuvent-ils oser demander plus? Cette seule réalité sociale - la création d'emplois - justifierait des conditions de travail en dessous de la loi. L'entreprise mobilise un argumentaire dans lequel elle demande au syndicat de faire passer «l'intérêt collectif » avant les droits individuels des travailleurs; pour autant, cet intérêt n'est pas autre chose que l'intérêt de l'entreprise. Dans cette situation, les syndicalistes se retrouvent responsables d'une situation où la menace de fermeture de l'entreprise est agitée par l'entreprise. Le syndicat a en quelque sorte dans ses mains la survie de l'entreprise et, selon l'argumentaire patronal, la survie même des ouvriers dans la mesure où le chômage est directement relié à l'augmentation de la criminalité. 
Dès lors, les travailleurs se trouvent face à un dilemme : soit ils acceptent de renoncer à leurs droits pour maintenir leur emploi, soit ils persévèrent dans leurs revendications au risque de perdre leur emploi. Lutter pour ses droits est, dans cette logique, le plus sûr moyen de perdre ses droits, ou du moins de les mettre en péril.

Les stratégies de délégitimation des revendications syndicales sont par ailleurs doublées de mécanismes de déresponsabilisation. Les arguments des employeurs visent ainsi à souligner dans quelle mesure leurs actions sont contraintes par l'extérieur : leurs politiques à l'égard des ouvriers ne relèveraient pas d'un choix mais d'une contrainte liée à des contextes sur lesquels les employeurs n'auraient pas de prise, dont la fragilité de l'entreprise dans le contexte de crise économique ou de la concurrence, surtout celle de l'économie chinoise.

\section{Des discriminations discrètes}

Une autre modalité de répression vise à mettre en doute l'éthique de l'organisation syndicale au nom de la recherche des gains collectifs, faisant peser le doute sur le désintéressement des actions syndicales et la réputation des syndicalistes. De nombreuses rumeurs circulent par exemple sur leurs agissements " intéressés » et sur la corruption et la moralité des dirigeants syndicaux. Des stratégies de division entre syndiqués et dirigeants passent notamment par des tentatives de récupération et de cooptation. Les employeurs proposent d'échanger la coopération des syndicalistes contre des rétributions financières ou contre des avantages sur le lieu de travail. Quand bien même les employés syndiqués ne s'adonneraient pas à ce genre de pratiques, l'objectif des employeurs est de discréditer auprès de la main-d'œuvre les syndicalistes corrompus, qui n'agiraient que pour leur propre compte et représentent une menace pour l'emploi. Ce sont le plus souvent les agents de maitrise et la direction des ressources humaines qui se chargent de ce travail de sape en diffusant des rumeurs et des commérages, en octroyant des bonus de production à certains travailleurs et en en refusant à d'autres parce qu'ils sont affiliés ou qu'ils sont proches de certains syndicalistes. Tout se passe comme si la proximité avec le syndicat devait être payée d'une manière ou d'une autre mais ceci de manière discrète. Lorsque ces agissements sont dénoncés par les syndicats, les chefs du personnel font valoir le fait qu'il ne s'agit pas de discriminations antisyndicales mais de décisions motivées par des considérations purement économiques liées à la productivité des salariés. Pour saisir la discrétion des discriminations, il faut bien comprendre qu'elles se réalisent de manière quotidienne, au cours d'actes routiniers et ordinaires : refus d'un bon de sortie pour aller à la sécurité sociale, refus de bonus de production, changement de postes, etc. Il arrive par ailleurs que les discriminations antisyndicales soient paradoxalement des discriminations positives. En effet, pour creuser un fossé entre les affiliés et le reste de la main-d'œuvre, la direction des ressources humaines octroie parfois des avantages aux syndicalistes (des sodas dans les bureaux de la direction, des avances sur salaires) puis font connaître ces avantages auprès d'autres travailleurs pour suggérer à nouveau l'étendue de la corruption syndicale.

\section{Le coût de l'activité syndicale}

Être syndicaliste n'implique pas seulement d'être victime de discriminations par les employeurs et parfois même de violence et de menaces de mort, cela a un coût tout 
simplement. En effet, le travail syndical, c'est-à-dire les sorties pour des cours de formation, des réunions ou encore des visites au Ministère du Travail et à l'inspection du travail, est régulé par un droit à des permis syndicaux, sorte d'autorisation d'absence, qui donnent droit à un salaire. Néanmoins, celui-ci ne comprend pas l'ensemble des bonifications, en quelque sorte la part variable du salaire contenue dans l'accomplissement d'heures supplémentaires et les bonus de production. Ce manque à gagner est souvent évoqué par les syndicalistes notamment quant à l'impact de leur pratique syndicale sur leur foyer. La rémunération des syndicalistes chute sensiblement, ce qui peut créer des conflits, qu'il s'agisse d'hommes ou de femmes. Voilà comment des syndicalistes de maquila évoquent les coûts financiers de leur activité syndicale :

- Adriana : « Du travail à ma chambre, de ma chambre au travail, le dimanche laver ou faire ce que je n'ai pas le temps de faire en semaine, je le fais le samedi et le dimanche, je n'ai pas le temps d'aller ne serait-ce qu'au parc [...]»

- Enquêteur : «Et les permis syndicaux ça change?»

- Adriana : "Oui, la vérité c'est que oui, travailler assis pendant douze heures, c'est fatiguant mais en apparence... Peut-être que les mains ne travaillent pas ni les pieds mais le cerveau oui donc oui mais les sorties m'aident, bien que cela m'affecte d'un point de vue économique parce qu'on a pas d'heures supplémentaires, pas de bonus de production.»

- Josué (23 ans, quatre années d'ancienneté, originaire de Chimaltenango) : « Ce qui est dur c'est au niveau économique. Quand on sort et on doit payer le transport, la nourriture, [...] Les photocopies aussi [...] Notre centrale nous a jamais parlé des coûts. » - Mario (28 ans, huit années d'ancienneté, originaire de Zacapa) : «Moi je perds, je ne sais pas, comme 200 quetzales sur mon salaire [soit l'équivalent de quatre journées de travail, soit 25 dollars], alors oui c'est dur. »

41 De plus, parce qu'ils sont souvent sollicités sur le lieu de travail, les syndicalistes se retrouvent souvent dans une situation où ils ne peuvent pas réaliser les objectifs de production. Ainsi, ils perdent de l'argent lors des sorties liées à l'activité syndicale mais aussi pendant les heures de travail.

\section{Les effets antisyndicaux de la violence ordinaire}

$42 \mathrm{Au}$ Guatemala, le climat de violence quotidienne a des effets sur l'activité syndicale. Le Guatemala détient l'un des taux d'homicides par habitants le plus élevé du monde avec un taux, dans les années 2000, proche de 45 homicides pour 100000 habitants (PNUD, 2010). Des groupes criminels organisés, les maras, ont pénétré les maquilas et y pratiquent des activités d'extorsion et de racket auprès des salariés et du personnel d'encadrement dans les usines. La maquila est en effet un terrain propice à l'accumulation de ressources pour ces groupes criminels. Dans une maquila de Mixco par exemple, l'ensemble des salariés devait payer vingt quetzales deux fois par mois aux maras sous peine de représailles auprès de leurs familles. Pour une usine employant près de 5000 ouvriers, les montants des extorsions pouvaient atteindre des montants très importants. Selon certaines estimations, les montants des extorsions organisées par ces groupes criminels auprès de commerces, de particuliers et d'employés de maquilas auraient atteint la somme de 100 millions de quetzales en 2005 (environ 13 millions de dollars) dont près de 11 millions de quetzales (environ 1,5 million de 
dollars) pour les seuls salariés des maquilas (Alvarez, 2010). Les maras tentent d'utiliser des syndicalistes pour les trafics au sein de l'usine, elles se servent concrètement de leurs réseaux auprès de la main d'œuvre ou du personnel d'encadrement pour essayer d'étendre leur influence dans les usines. Ainsi, les maras menacent les syndicalistes de représailles s'ils ne participent pas aux extorsions dans l'usine. Ces tentatives d'instrumentalisation des leaders syndicaux paralysent l'activité syndicale dans la mesure où les syndicalistes menacés doivent parfois quitter temporairement leur travail pour échapper aux menaces. Les employeurs tentent de lutter contre les maras à travers des campagnes de démantèlement organisées depuis le Ministère de l'Intérieur (Ministerio de Gobernación). Mais, à de nombreuses reprises, ces opérations anti maras, ont provoqué licenciements collectifs de salariés soupçonnés de participer aux trafics, permettant de mettre en pratique le licenciement des syndiqués et donc de porter un coup à l'organisation syndicale.

\section{Conclusion}

L'analyse des conditions concrètes d'organisation et de pérennisation de l'action syndicale dans les zones franches d'exportation guatémaltèques souligne une variété d'obstacles. Au-delà des contraintes juridiques, l'exercice des droits syndicaux est limité de fait par un certain nombre de pratiques illégales de la part des employeurs ainsi que par les difficultés que rencontrent les organisations syndicales nationales pour mobiliser les salariés, le plus souvent femmes, des maquilas. De plus, l'environnement social particulièrement violent de la société guatémaltèque, pouvant être qualifié de criminel, s'ajoute à la répression antisyndicale devenue la norme, plutôt que l'exception, sous forme de discriminations variées, ingérences, menaces, pour ne pas parler de stratégies de contournement des lois ...

Malgré les alternances politiques et les pressions internationales menées par des mouvements d'activistes et par des organisations internationales, le contexte de mobilisation ainsi que l'exercice des droits syndicaux au Guatemala restent particulièrement défavorables. Une récente étude de la confédération syndicale internationale parue en 2014 place même le Guatemala parmi les pires pays du monde avec la Colombie, le Zimbabwe, Swaziland and Myanmar (ITUC, 2014). Les nombreux cas de violations manifestes des droits - comme les assassinats de syndicalistes ou encore les dossiers de licenciements illégaux de travailleurs des usines de maquilas aboutissent rarement.

\section{BIBLIOGRAPHIE}

Alvarez, Lorena, « Economia ilegal : el diez por ciento del PIB », El Periódico, 29 janvier 2010.

Anner, Mark, « Two Logics of Labor Organizing in the Global Apparel Industry », International Studies Quarterly, vol. 53, n 3, 2009, p. 545-570. 
Armbruster-Sandoval, Ralph, Globalization and Cross-Border Labor Solidarity in the Americas : The Anti-Sweatshop Movement and the Struggle for Social Justice, New York, Routledge, 2005.

Bandy, Joe, « Paradoxes of Transnational Civil Societies under Neoliberalism : The Coalition for Justice in the Maquiladoras ", Social Problems, vol. 51, n 3, 2004, p. 410-431.

Carty, Victoria, « Transnational Labor Mobilizing in Two Mexican Maquiladoras : The Struggle for Democratic Globalization », Mobilization : an International Journal, vol. 9, n 3, 2004, p. 295-310.

Centro internacional para investigaciones en derechos humanos, La ley de maquila, Guatemala Ciudad, CIIDH, 2006.

Delpech, Quentin, Mobilisations syndicales et violence au sud. Protester dans les usines de la soustraitance internationale au Guatemala, Paris, Karthala, 2014.

ENEI, Encuesta Nacional de Empleo e Ingresos (Guatemala), 2010.

Frundt, Henry, « Movement Theory and International Labor Solidarity », Labor Studies Journal, vol. $30, n^{\circ} 2,2005$, p. 19-40.

Frundt, Henry, Refreshing Pauses : Coca Cola and Human rights in Guatemala, New York, Praeger, 1987.

Gereffi, Gary, «The Transformation of the North American Apparel Industry : Is NAFTA a Curse or a Blessing? ", Integration and Trade, vol. 4, n 11, 2000, p. 46-95.

Gopalakrisnan, Ramapriya, Freedom of association and collective bargaining in export processing zones : Role of the ILO supervisory mechanisms, document de travail n, Genève, BIT, 2007.

International Trade Union Confederation, Countries at risk : 2013 Report on Violations of Trade Union Rights, ITUC, 2014.

Levenson-Estrada, Deborah, Trade Unionists Against Terror : Guatemala City (1954-1985), Chapel Hill, University of North Carolina Press, 1994.

McKay, Steven, Satanic Mills or Silicon Islands? The Politics of High-Tech Production in the Philippines, Ithaca, Cornell University Press, 2006.

McKay, Steven, Securing Commitment in an Insecure World : Power and the Social Regulation of Labor in the Philippine Electronics Industry, Ph.D. dissertation, Department of Sociology, University of Wisconsin, 2001

Médecins du Monde, « Maquilas : bienvenue en zone de non droit », 2010

OIT, Rapport de la Commission de l'application des normes de la Conférence internationale du Travail, Genève, OIT, 2011.

OIT, Rapport de la Commission de l'application des normes de la Conférence internationale du Travail, Genève, OIT, 2009.

OIT, La Libertad Sindical y la Negociación Colectiva en Centroamérica, Panamá y República Dominicana : Un Panorama de su estado Actual, San José, OIT, 2009.

Pena, Devon, The Terror of the machine. Technology, Work, Gender and the Ecology on the U.S.-Mexico Border, Austin, University of Texas Press, 1997.

Perez Sainz Juan Pablo, From the Finca to the Maquila : Labor and Capitalist Development in Central America, Boulder, Westview Press, 2000. 
Perez-Sainz, Juan Pablo, « Guatemala : The Two Faces of Metropolitan Area » in Alejandro Portes et al., The Urban Caribbean : Transition to the New Global Economy, Baltimore, The Johns Hopkins University Press, 2007, p. 124-154.

Perez Sainz Juan Pablo, Leal, Angela, Pequena empresa, capital social y ethnicidad : el caso de San Pedro Sacatepéquez, Guatemala, FLACSO, 1992.

Petersen Kurt, The Maquiladora Revolution in Guatemala, Yale, Orville H. Schell Jr Center for International Human Rights, 1992.

PNUD, Informe nacional de desarrollo humano (2009-2010), Guatemala, PNUD, 2010.

Rodriguez-Garavito, Cesar, « Sewing Resistance : Transnational Organizing, Anti Sweatshop Activism, and Labor Rights in the US Caribbean Basin Apparel Industry (1990-2005) », Working Paper Series, The Center for Migration and Development, Princeton University, 2008.

VESTEX, « Perfil de la industria », http://vestex.com.gt/, page visitée en mars 2010 (le document n'est plus disponible).

Williams, Heather, « Mobile Capital and Transborder Labor Rights Mobilization », Politics \& Society, vol. 27, n 1, 1999, p. 139-166.

\section{NOTES}

1. Pour un aperçu des publications de l'OIT sur le thème de l'emploi et des conditions d'exercice des droits fondamentaux des travailleurs dans les zones franches d'exportation à travers le monde, voir le site de l'Organisation internationale du Travail: http://www.ilo.org/public/ english/support/lib/resource/subject/epzbibl.pdf

2. Dans le bassin des Caraïbes, cette activité économique employait ainsi au milieu des années 1990 près de 235000 travailleurs. À la fin des années 1990, 51 \% des importations américaines depuis l'Amérique centrale impliquaient des produits issus de cette industrie. Néanmoins, depuis 2004, le secteur a subi la concurrence d'industries d'autres pays, notamment de la Chine, et de très nombreux emplois ont été détruits.

3. Selon le rapport 2009 de la commission d'experts, "Quant à l'affiliation syndicale, d'après les autorités administratives, il existe six syndicats auxquels sont affiliés 562 travailleurs des maquilas, alors que le secteur compte près de 200000 travailleurs. Pour la direction du mouvement syndical, il n'existe que deux syndicats dans ce secteur. Quel que soit le chiffre exact, dans les maquilas, l'activité syndicale reste faible et la négociation collective peu répandue, et un problème d'application des conventions numéros 87 et 98 se pose. » Voir (OIT, 2009).

4. Il reste en effet à réaliser une enquête approfondie sur les profils des salariés des ZFE au Guatemala, c'est-à-dire des données précises et fiables sur l'âge, les origines ethniques, le sexe, les trajectoires d'emploi antérieures et postérieures, les origines géographiques des travailleurs et des travailleuses.

5. Il existe depuis 2007 trois types de salaires minimums : un pour les activités agricoles, un pour les activités non agricoles et, enfin, un pour les activités de maquila et d'exportation.

6. La canasta básica vital correspond à l'ensemble des biens et des services essentiels pour satisfaire les nécessités basiques pour le bien-être d'une famille. Selon les données officielles, la CBV se compose des dépenses liées à l'alimentation, aux vêtements, à l'habitation, au mobilier, à la santé, à l'éducation, à la culture, aux loisirs.

7. La canasta básica alimentaria correspond à l'ensemble de la quantité de consommation quotidienne d'une famille de 5,38 personnes comprenant les aliments suivants: viande, œufs, haricots, maïs, céréales, sucres, légumes, fruits, laitages et autres produits basiques. 
8. Les usines SAE-A ont environ dix années d'ancienneté.

9. Néanmoins, ces pratiques sont possibles dans la mesure où les « foyers » d'organisation, ou dit autrement, les leaders ou les fortes têtes, sont identifiés préalablement. Cette identification est rendue possible par le travail des chefs de personnel auxquels il est demandé de porter une attention particulière aux rumeurs d'organisation. Dans certains cas, les chefs de personnel peuvent même faire appel à des informateurs au sein de la main-d'œuvre. Pour prévenir l'émergence de contestation organisée, les employeurs procèdent par ailleurs à une rotation importante de la main-d'œuvre.

10. Extrait du procès-verbal de la réunion de conciliation entre le syndicat SITRASAE et les représentants de l'entreprise SAE-A International (08/07/07).

\section{RÉSUMÉS}

Cet article tente de cerner les obstacles d'une part à la syndicalisation et d'autre part à la consolidation des organisations syndicales dans les usines de l'habillement du Guatemala, appelées maquilas. Il tente de cerner les obstacles pratiques et les contraintes quotidiennes à l'exercice des droits syndicaux des salariés. Ce travail se fonde sur un travail d'enquête approfondi, mené entre 2006 et 2009, dans quatre usines de l'habillement de la proche périphérie de Guatemala Ciudad où ont émergé depuis le début des années 2000 quatre syndicats d'employés.

This paper describes the barriers to labor organizing on the one hand and to the union activity on the other hand, in the export-oriented apparel industry of Guatemala, the so-called maquilas. It identifies the practical barriers and everyday constraints to the defense of labor rights in those factories. This work is based on an in-depth fieldwork research undertaken between 2006 and 2009 in four maquilas in the outskirt of Guatemala City, where unions managed to emerge in the early 2000 s.

Este artículo describe los obstáculos a la sindicalización por un lado y a la consolidación de las organizaciones sindicales por otro lado en las fábricas del vestuario de Guatemala, las famosas «maquilas». Se identifican los obstáculos prácticos y las coacciones cotidianas en contra de los derechos laborales en estas fábricas. Este trabajo se basa en una investigación entre 2006 y 2009 en cuatros fábricas de vestuario en la cercanía de Guatemala Ciudad, donde se formaron organizaciones sindicales al principio de los años 2000.

\section{INDEX}

Palabras claves : Guatemala, sindicalismo, repression, fabricas, subcontratación, obreros, vestuario

Mots-clés : Guatemala, syndicalisme, répression, usine, sous-traitance, ouvriers, habillement

Keywords : Guatemala, labor unionism, repression, plant, outsourcing, workers, apparel 
AUTEUR

QUENTIN DELPECH

Quentin Delpech est docteur en science politique de l'université Panthéon Sorbonne Paris 1 et chercheur associé au centre d'études mexicaines et centraméricaines (CEMCA). Il est spécialiste des mouvements sociaux au Guatemala. quentdelpech@yahoo.fr. 